\title{
The impact of H. pylori and/or T. gondii infection on recurrence of gastritis and gastric ulcer
}

\author{
Original \\ Article

\begin{abstract}
Departments of Pathology (Microbiology Unit) ${ }^{1}$, Microbiology and Immunology ${ }^{2,5}$, Medical Parasitology ${ }^{3}$, and Internal Medicine ${ }^{4}$, College of Medicine, Jouf University, Sakaka, AlJouf, Saudi Arabia ${ }^{1}$, Faculties of Medicine ${ }^{2-5}$, Kafrelsheikh ${ }^{2,4}$, Benha $^{3}$, and Mansoura ${ }^{5}$ Universities, Kafrelsheikh, Qalyubia, and Dakahlia Governorates, Egypt
\end{abstract} \\ Amany A Ghazy ${ }^{1,2}$, Waleed E Elawamy ${ }^{1,3}$, Tamer Haydara ${ }^{4}$, Ahmed E Taha ${ }^{1,5}$
}

\begin{abstract}
Background: Concomitant H. pylori and T. gondii infections are two widespread diseases transmitted by fecal-oral route. Several studies documented association of both infections in gastritis and peptic ulcer diseases.

Objective: The aim of this work is to evaluate the relation between recurrent gastritis or gastric ulcers and coinfection with $H$. pylori and T. gondii.

Subjects and Methods: Investigations included upper gastrointestinal tract (GIT) endoscopic biopsy, detailed personal questionnaire, serum detection of anti-cag A and anti-vac A for H. pylori, anti-Toxoplasma IgM and IgG antibodies. Selected cases were from patients attending the Gastroenterology and Hepatology Department of Kafrelsheikh University Hospital. In total, 80 participants were distributed as 32 naïve cases, 28 recurrent and 20 with no gastric infections as control. For diagnosis of $H$. pylori, sandwich ELISA immunoassay (EIA) test was performed to detect $H$. pylori surface coproantigen in fresh stools taken after endoscopy. In patients' serum samples, IgG for H. pylori cag-A and vac-A antigens, IgM and IgG for T. gondii were detected by ELISA.

Results: Recurrence of $H$. pylori infection increased in age groups 21-30 and 31-40 years, mainly in males and in those eating junk foods. Severe gastric ulcers appeared in combined H. pylori and T. gondii infections. Recurrent cases showed $71.4 \%$ positive $H$. pylori anti-cag A, $57.1 \%$ H. pylori anti-vac A, 35.7\% both $H$. pylori antigens, 57.1\% anti-Toxoplasma IgM, and 71.4\% anti-Toxoplasma IgG, 35.7\% concomitant bacterial and parasitic infection. High IgM positivity rate was recorded in recurrent cases while IgG was recorded in naïve cases.

Conclusion: Toxoplasmosis when combined with H. pylori participates in recurrence of the latter, causing higher severity of gastritis and gastric ulcer. Eating junk foods and being middle aged are contributing risk factors.
\end{abstract}

Keywords: coproantigens; gastritis; gastric ulcers; H. pylori; T. gondii; recurrence.

Received: 15 September, 2021, Accepted: 20 November, 2021.

Corresponding Author: Waleed E. Elawamy, Tel.: +966 590997875, E-mail: waleedelawamy2015@yahoo.com

Print ISSN: 1687-7942, Online ISSN: 2090-2646, Vol. 14, No. 3, December, 2021.

\section{INTRODUCTION}

The association of the two widespread infectious diseases caused by the gram negative bacterium, $H$. pylori, and the apicomplexan parasite, T. gondii, was studied in gastritis and peptic-ulcer formation ${ }^{[1]}$. This positive association may occur by the fecal-oral route from cat feces. High detection rates of $H$. pylori in cat feces raised the possibility of zoonotic transmission of both pathogens ${ }^{[2]}$.

Treatment of $H$. pylori infection markedly decreases the occurrence of peptic ulcers. It was found that gastric ulcers tend to recur more than duodenal ulcers usually at the same original site ${ }^{[3]}$. The most common complication associated with peptic ulcer disease is peptic ulcer bleeding. Thus understanding the role of $H$. pylori in the pathogenesis of this bleeding is crucial to prevent upper GIT hemorrhage ${ }^{[4]}$. Of note, $30 \%$ of $H$. pylori infected patients present by mild to severe upper GIT diseases such as gastritis, peptic ulcer, gastric cancer or mucosa-associated lymphoma in spite of having been infected during childhood or being asymptomatic for a long period ${ }^{[5]}$.

Combination of $H$. pylori infection with $A$. lumbercoides and T. gondii altered $H$. pylori gastritis in rodents' models. Elevation of IgE and antiinflammatory Th2-IgG1 responses to $H$. pylori was found to correlate with $A$. lumbercoides IgG antibodies detection. Reduction of Th1-IgG2, IgG3 and IgG4 responses to $H$. pylori correlated with high $T$. gondii titres $^{[6]}$.

Possession of a well-known virulence factor as the cytotoxin-associated gene A (cag-A), allows some $H$. pylori strains to cause more severe inflammation and widespread atrophy in gastric mucosa than those lacking this gene ${ }^{[7,8]}$. Early immunological detection of cag-A protein just after $H$. pylori infection 
by determination of specific gastric mucosal IgA and serum IgG antibodies marks the cag-A-positive strains as the pathogenic and immune dominant agent in cases of gastric ulcers ${ }^{[9]}$. A significant association of vac-A genotypes $m 1, s 1 m 1$ and $s 2 m 1$, as virulence factors for peptic ulcer, was observed in $H$. pylori infected patients $^{[10]}$.

On the other hand, bidirectional endoscopy in an immunocompromised patient with disseminated toxoplasmosis, revealed large ulcerations in the gastric cardia and fundus with severe active gastritis and ischemic changes ${ }^{[11]}$. This was attributed to a strong immune response controlled by a series of regulatory mechanisms against Toxoplasma. The disturbed balance between CD4 and CD8 cells, and the immunosuppression mediated by HLA-DR molecules is enhanced, which may lead to gastritis and diarrhoea ${ }^{[12]}$.

Manifestations of chronic infections with $T$. gondii and/or Helicobacter spp. probably depend on the host immune response and patients who suffer from chronicity were found to carry more than one pathogen ${ }^{[13]}$. Although the investigators were unable to explain how multiple active infections interact with each other or how they affect the disease prognosis, they claimed that concomitant infection by $T$. gondii and $H$. felis was found to enhance the susceptibility of mice to $H$. felis infection ${ }^{[13]}$.

This was associated with significant elevation of gastric mucosal IFN- $\gamma$ and IL-12, considerable reduction of IL-10, severe gastric mucosal inflammation, loss of parietal cells, atrophy, and metastatic cell changes. Consequently, the aim of the present work was to evaluate the relation between recurrent gastritis and/ or gastric ulcers and coinfection with $H$. pylori and $T$. gondii.

\section{SUBJECTS AND METHODS}

This case-control study was conducted during the period from January 2020 to July 2020. Stool and serum samples were obtained from patients in the Gastroenterology and Hepatology Departments, Kafrelsheikh University Hospital. Samples were transferred to Parasitology and Microbiology Departments, Faculty of Medicine, Kafrelsheikh University for parasitological and microbiological investigations.

Study design: Patients complaining of sever upper GIT symptoms were regarded as eligible for endoscopy, and stool analysis for $H$. pylori coproantigens. Those with normal endoscopic findings and negative for $H$. pylori coproantigens were considered as apparently healthy controls. Patients with endoscopic findings and positive for $H$. pylori coproantigens who presented for the first time were considered as naïve cases; and those who returned after one year because of persistent symptoms were considered as recurrent cases. All cases were subjected to $H$. pylori and T. gondii analysis by sandwich ELISA immunoassay (EIA) for coproantigen detection in stool specimens and by ELISA for serum antibodies against $H$. pylori and T. gondii.

Participants: History was recorded from patients including their type of diet. Patients (no.=60) found eligible for upper GIT endoscopy came to the hospital complaining of epigastric pain, heartburn, abdominal discomfort and/or vomiting, not improved by empirical anti-acids or proton pump inhibitors alone. The inclusion criteria included naïve $H$. pylori infected patients (no. $=32$ ) having upper GIT symptoms for the first time and confirmed diseased by endoscopy. Recurrent H. pylori patients (no.=28) were those complaining of the same symptoms within one year. Choice of apparently healthy controls (no.= 20) depended on the endoscopic examination (pink, smooth and lustrous gastric mucosa). The exclusion criteria included patients who had taken anti $H$. pylori therapy four weeks before endoscopy, or had any gastro-intestinal, hepatic, or renal diseases. Fresh stool and serum samples were collected from each participant after endoscopy.

H. pylori surface coproantigen (HpSA) test: A fresh stool sample from each participant was collected and stored at $-20^{\circ} \mathrm{C}$ until analysis. Detection of $\mathrm{HpSA}$ in stools was by EIA for antigen detection according to the manufacturers' instructions ${ }^{[14]}$ (Premier Platinum HpSA, Meridian Bioscience Inc, USA).

Antibody assay against $H$. pylori coproantigens: Serum samples were screened for $H$. pylori antibodies (anti-cag-A IgG, and anti-vac-A IgG) by ELISA ${ }^{[15]}$ (Xingkang Company, Shenzhen, China). The cut off values were determined for both. The results were interpreted by the positions of the matrices and the mean grey levels of the spots in the matrices.

Detection of anti-Toxoplasma IgM and IgG in patients' sera: Serum samples were screened using ready-made ELISA kits (Abcam, USA), and an ELISA plate reader (Bio-Rad, Hercules, CA). The optical density was measured at $450 \mathrm{~nm}$ and the antibody (IgM and IgG) titre for each sample was calculated ${ }^{[16]}$ Following the manufacture's protocol, a Toxoplasma IgM titre $<0.8$ was considered negative; $0.8-1.0$ was considered equivocal; and $>1$ was considered positive. A Toxoplasma IgG titre $<5$ was considered negative; 5-7 was equivocal and $>7$ was positive.

Study groups: According to the results of stool and serum investigations, as well as endoscopy, patients were categorized into 8 groups as shown in the following table. 


\begin{tabular}{|c|c|c|}
\hline $\begin{array}{c}\text { Groups } \\
\text { (No. of cases) }\end{array}$ & Groups identity & Characteristics \\
\hline $1(4)$ & Infected, sero-negative group & $\begin{array}{l}\text { Patients positive for } H \text {. pylori antigen in stools and serologically } \\
\text { negative in all serum samples. }\end{array}$ \\
\hline $2(4)$ & H. pylori, cag-A group & Patients positive for anti-cag-A IgG antibodies only. \\
\hline $3(2)$ & H. pylori, vac-A group & Patients positive for anti-vac-A IgG antibodies only. \\
\hline $4(2)$ & Both H. pylori antigens group & Patients positive for both anti-cag-A and anti-vac-A IgG antibodies. \\
\hline $5(4)$ & Recently acquired toxoplasmosis group & Patients positive for anti-Toxoplasma IgM antibodies only. \\
\hline $6(6)$ & Chronic (latent) toxoplasmosis group & Patients positive for anti-Toxoplasma IgG antibodies only. \\
\hline $7(38)$ & Concomitant infections group & $\begin{array}{l}\text { Patients positive for concomitant anti-cag-A, anti-vac-A, anti- } \\
\text { Toxoplasma IgM, or anti-Toxoplasma IgG antibodies. }\end{array}$ \\
\hline $8(20)$ & Controls & $\begin{array}{l}\text { Patients having symptoms, with healthy endoscopic gastric mucosa, } \\
\text { negative } H \text {. pylori coproantigens, and positive Toxoplasma IgG only. }\end{array}$ \\
\hline
\end{tabular}

Ethical approval: The study was approval by Ethical Committee of Faculty of Medicine, Kafrelsheikh University, 2019. We followed the guidelines as per the Declaration of Helsinki for involving human participants. Permissions in the form of informed consents were taken from the participants. All patients and control subjects were informed of the investigations results.

Statistical analysis: Data were tabulated and analysed using SPSS version 23. Demographic data were presented as categorical groups, and serological tests titres were presented as mean \pm standard deviation. Data for categorical variables was expressed as percentages. Differences between variables were compared by using chi-square analysis $\left(X^{2}\right)$. Fisher exact test was used if the expected count was less than 5. $P$ value $<0.05$ was considered as statistically significant relationship between variables.

\section{RESULTS}

Recurrence of gastric ulcer: In the 28 cases with recurrent gastritis the highest prevalence was in the age groups 21-30 years and 31-40 years with the same percentage $(42.9 \%)$. In the 32 naïve cases, the prevalence was in the 21-30 years age group (37.5\%). Both were statistically significant $(P<0.05)$. Regarding the gender, recurrence in males $(71.4 \%)$ was significantly $(P<0.05)$ more than in females $(28.6 \%)$. In both recurred and naïve cases distribution by diet habits showed insignificant prevalence in junk foods compared to home and fat diets (Table 1).

Correlation of gastritis severity and the type of infection: Endoscopic examination categorized fundus findings as mild, moderate, and severe gastritis. Severe gastritis (erosions and ulcers) ranged from erosive fundal gastritis to severe pan gastric congested mucosa with hemorrhagic fundal gastritis (Figure 1d).

Table 1. Patients complaining of naïve and recurrent gastric problems in relation to age, sex and diet.

\begin{tabular}{|c|c|c|c|c|c|}
\hline & \multirow{2}{*}{$\begin{array}{c}\text { Control }(n=20) \\
\text { No. }(\%)\end{array}$} & \multirow{2}{*}{$\begin{array}{c}\text { Naïve cases }(n=32) \\
\text { No. }(\%)\end{array}$} & \multirow{2}{*}{$\begin{array}{c}\text { Recurrent cases }(n=28) \\
\text { No. }(\%)\end{array}$} & \multicolumn{2}{|c|}{ Statistical analysis } \\
\hline & & & & $X^{2}$ & $P$ value \\
\hline \multicolumn{6}{|l|}{ Age } \\
\hline $11-20$ & $6(30.0)$ & $6(18.8)$ & $0(0.0)$ & \multirow{5}{*}{ 14.36@ } & \multirow{5}{*}{$<0.05^{*}$} \\
\hline $21-30$ & $7(35.0)$ & $12(37.5)$ & $12(42.9)$ & & \\
\hline $31-40$ & $5(25.0)$ & $6(18.8)$ & $12(42.9)$ & & \\
\hline 41-50 & $2(10.0)$ & $6(18.8)$ & $4(14.3)$ & & \\
\hline $51-60$ & $0(0.0)$ & $2(6.3)$ & $0(0.0)$ & & \\
\hline \multicolumn{6}{|l|}{ Sex } \\
\hline Male & $11(55.0)$ & $20(62.5)$ & $20(71.4)$ & \multirow[t]{2}{*}{1.339} & \multirow[t]{2}{*}{$>0.05$} \\
\hline Female & $9(45.0)$ & $12(37.5)$ & $8(28.6)$ & & \\
\hline \multicolumn{6}{|l|}{ Diet } \\
\hline Home & $13(65.0)$ & $16(50.0)$ & $8(28.6)$ & \multirow{3}{*}{8.53} & \multirow{3}{*}{$<0.05^{*}$} \\
\hline Junk & $5(25.0)$ & $12(37.5)$ & $18(64.3)$ & & \\
\hline Increased fats diet & $2(10.0)$ & $4(12.5)$ & $2(7.1)$ & & \\
\hline
\end{tabular}


Severity of endoscopic findings in G7 associated with concomitant infection by $H$. pylori and T. gondii was in $18 / 60(30 \%)$ of all patients and in 18/18 (100\%) of severe cases. Moderate gastritis observed as moderate antral gastritis, moderate fundal gastritis, granular mucosa of the body or congested erythematous body with longitudinal erosions (Fig. 1b and c), appeared to be higher in concomitant infections 12/16 (75\%). Mild gastritis that ranged from mild antral erythematous mottling to antral gastritis with duodeno-gastric biliary reflux (Figure 1a) occurred in 8/26 (30.8\%) of concomitant infections (G7), and in 6/26 (23.1\%) of chronic latent toxoplasmosis (G6). Total moderate and mild cases of gastritis represented 16/60 (26.7) and $26 / 60(43.3 \%)$ of all patients respectively. Relation between all variables was statistically significant $(P<$ 0.001), (Table 2).

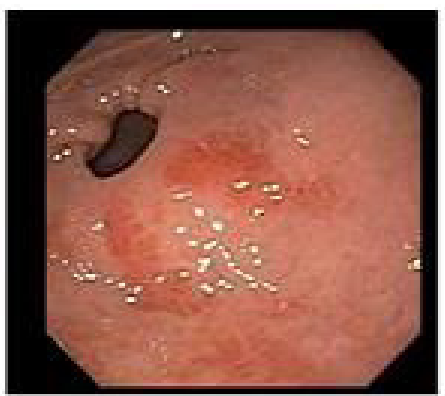

(a)

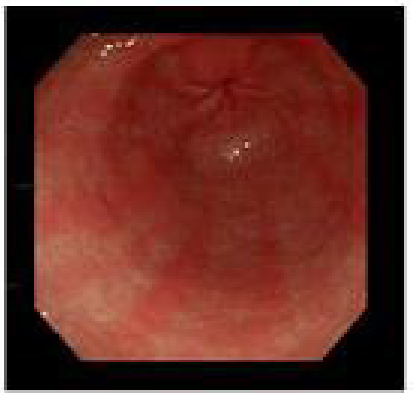

(b)

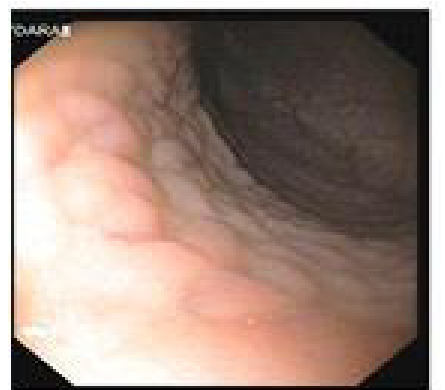

(c)

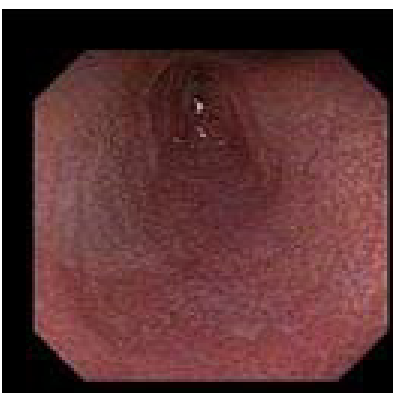

(d)

Fig. 1. Endoscopic findings of different degrees of gastritis severity in patients with $H$. pylori infections and/or toxoplasmosis. (a) Patchy antral gastritis, erythematous mottling. (b) Moderate antrum gastritis, erythematous mottling. (c) Granular mucosa (body). (d) Pan-gastritis, erythematous mottling.

Table 2. Severity of gastritis in the different groups.

\begin{tabular}{|c|c|c|c|c|c|c|}
\hline \multirow[b]{2}{*}{ Groups } & \multicolumn{4}{|c|}{ Severity of gastritis (Endoscopy) } & \multicolumn{2}{|c|}{ Statistical analysis } \\
\hline & $\begin{array}{c}\text { Mild } \\
\text { No. (\%) }\end{array}$ & $\begin{array}{c}\text { Moderate } \\
\text { No. (\%) }\end{array}$ & $\begin{array}{c}\text { Severe } \\
\text { No. (\%) }\end{array}$ & Total & Fisher exact test & $P$ value \\
\hline 1 & $4(15.4)$ & $0(0)$ & $0(0)$ & $4(6.7)$ & \multirow{7}{*}{$\begin{array}{c}29.41 \\
<0.001\end{array}$} & \\
\hline 2 & $2(7.7)$ & $2(12.5)$ & $0(0)$ & $4(6.7)$ & & \\
\hline 3 & $0(0)$ & $2(12.5)$ & $0(0)$ & $2(3.3)$ & & \\
\hline 4 & $2(7.7)$ & $0(0)$ & $0(0)$ & $2(3.3)$ & & \\
\hline 5 & $4(15.4)$ & $0(0)$ & $0(0)$ & $4(6.7)$ & & \\
\hline 6 & $6(23.1)$ & $0(0)$ & $0(0)$ & $6(10)$ & & \\
\hline 7 & $8(30.8)$ & $12(75)$ & $18(100)$ & $38(63.3)$ & & \\
\hline Total & $26(43.3)$ & $16(26.7)$ & $18(30.0)$ & $60(100)$ & & \\
\hline
\end{tabular}

Table 3. H. pylori and T. gondii infections in cases with naïve and recurrent gastric problems.

\begin{tabular}{|c|c|c|c|c|c|c|}
\hline \multirow{2}{*}{ Type of test } & \multirow{2}{*}{ Test result } & \multirow{2}{*}{$\begin{array}{c}\text { Control }(n=20) \\
\text { No. }(\%)\end{array}$} & \multirow{2}{*}{$\begin{array}{c}\text { Naïve cases }(n=32) \\
\text { No. }(\%)\end{array}$} & \multirow{2}{*}{$\begin{array}{c}\text { Recurrent cases }(n=28) \\
\text { No. }(\%)\end{array}$} & \multicolumn{2}{|c|}{ Statistical analysis } \\
\hline & & & & & $X^{2}$ & $P$ value \\
\hline \multirow{2}{*}{ H. pylori cag-A } & Positive & $0(0.0)$ & $8(25.0)$ & $20(71.4)$ & \multirow{2}{*}{28.51} & \multirow{2}{*}{$<0.001^{*}$} \\
\hline & Negative & $20(100.0)$ & $24(75.0)$ & $8(28.6)$ & & \\
\hline \multirow{2}{*}{ H. pylori vac-A } & Positive & $0(0.0)$ & $14(43.7)$ & $16(57.1)$ & \multirow{2}{*}{17.14} & \multirow{2}{*}{$<0.001^{*}$} \\
\hline & Negative & $20(100.0)$ & $18(56.3)$ & $12(42.9)$ & & \\
\hline \multirow{2}{*}{ Toxoplasma IgM } & Positive & $0(0.0)$ & $4(12.5)$ & $16(57.1)$ & \multirow{2}{*}{17.63} & \multirow{2}{*}{$<0.001^{*}$} \\
\hline & Negative & $20(100.0)$ & $28(87.5)$ & $12(42.9)$ & & \\
\hline \multirow{2}{*}{ Toxoplasma IgG } & Positive & $6(30.0)$ & $26(81.3)$ & $20(71.4)$ & \multirow{2}{*}{11.02} & \multirow{2}{*}{$<0.05^{*}$} \\
\hline & Negative & $14(70.0)$ & $6(18.7)$ & $8(28.6)$ & & \\
\hline
\end{tabular}


Relation between $H$. pylori positive cases and the presence of virulent factors, cag-A or vac- $A$ antibodies: $H$. pylori infected cases with cag-A positive versus negative status were 28/60 (46.67\%) and $32 / 60(53.33 \%)$ respectively. On the other hand, $H$. pylori cases with vac-A positive versus negative status were 30/60 (50\%) for each group of cases, showing statistical insignificance $(P>0.05)$ (Table 4$)$.
Relation of Toxoplasma antibody positivity in naïve and recurrent gastritis cases: The difference in IgM mean values in naïve $(0.90+1.50)$ versus recurrent cases $(2.89+2.34)$ was statistically significant $(P<0.0001)$. The difference in IgG mean values which were on the whole positive in both naïve (30.68 \pm 17.43$)$ and recurrent $(14.60 \pm 9.65)$ cases was statistically insignificant (Table 5).

Table 4. H. pylori positive virulent factors cag-A and vac-A status.

\begin{tabular}{llccccc}
\hline \hline & & \multicolumn{3}{c}{ H. pylori cag-A } & \multicolumn{2}{c}{ Statistical analysis } \\
\cline { 3 - 6 } & & Negative & Positive & Total & $\boldsymbol{X}^{2}$ & \multicolumn{2}{c}{$\boldsymbol{P}$ value } \\
\hline \multirow{3}{*}{ H. pylori vac-A A } & Negative & $14(46.67 \%)$ & $16(53.33 \%)$ & 30 & \multirow{2}{*}{$>07$} & $>05^{*}$ \\
& Positive & $18(60 \%)$ & $12(40 \%)$ & 30 & 60 & \\
& Total & $32(53.33 \%)$ & $28(46.67 \%)$ & 6 & & \\
\hline \hline
\end{tabular}

*: Insignificant.

Table 5. Toxoplasma mean IgG and IgM antibody values in naïve and recurrent gastric problems.

\begin{tabular}{lcccc}
\hline \hline & Control $(\mathbf{n = 2 0})$ & Naïve cases $(\mathbf{n = 1 6 )}$ & Recurrent cases $(\mathbf{n = 1 4 )}$ & Statistical analysis \\
\cline { 2 - 5 } & Mean $\pm \mathbf{S D}$ & Mean $\pm \mathbf{S D}$ & Mean \pm SD & $\boldsymbol{P}$ value \\
\hline Toxoplasma IgM & $0.39 \pm 0.36$ & $0.90 \pm 1.50$ & $2.89 \pm 2.34$ & $<0.001^{*}$ \\
Toxoplasma IgG & $6.78 \pm 7.75$ & $30.68 \pm 17.34$ & $14.60 \pm 9.65$ & $>0.05$ \\
\hline
\end{tabular}

Toxoplasma IgM evaluation: Negative: $<0.8$, Equivocal: 0.8-1; Positive: $>1$; Toxoplasma IgG evaluation: Negative: $<5$, Equivocal: 5-7 Positive: > 7. *: Significant $(P$ value $<0.05)$.

\section{DISCUSSION}

Several virulence factors including those of $H$. pylori constitute high risks for severe gastritis. A recent study encouraged researchers to perform more multidisciplinary efforts to understand the virulence factors of gastric complications ${ }^{[17,]}$. Gale et al.$^{[1]}$ reported that $H$. pylori and latent toxoplasmosis positive individuals were more liable to cognitive deficiency because of gastritis and vitamin B12 deficiency. This encouraged us to investigate the outcome of these two infections on recurrent gastritis.

In our study evaluation of factors that may influence the recurrence of gastric ulcers revealed that the most affected age groups were 21-30 years and 31-41 years as compared to previously reported mean age of 58.2 years old, with occurrence of peptic ulcer in $29.6 \%$ of patients over 70 years of age ${ }^{[18]}$. In another report, benign gastric ulcer (BGU) recurrence after eradication of $H$. pylori was recorded at the mean age of $65.3 \pm 15.2$ years, while in the non-recurrent group, the mean age was $49.1 \pm 10.9$ years $^{[19]}$. Additionally, we noted that recurrence among males was significantly higher (71.4\%; $\mathrm{P}<0.05)$ than in females $(28.6 \%)$ which is in accordance with the stated $70.3 \%$ of peptic ulcers in males $^{[19]}$.

According to Mahmood et al. ${ }^{[20]}$, there was significant association between the increased prevalence of $H$. pylori infection and eating food from street vendors, while cases who denied that and preferred eating fruits, showed no associated infection. This agrees with our results concerning the highest percentage of recurrence (64.3\%) among all recurrent cases who commonly eat junk foods. A similar report concerning individuals consuming junk food showed high prevalence of $H$. pylori infection with strong association to acid peptic disease in $157 / 200$ males and $142 / 200$ females $^{[21]}$. Accordingly, it was recommended that lipids intake in diets, without concentration of saturated fats, should be adjusted to protect against peptic ulcers ${ }^{[22]}$.

The current study revealed that in G7 patients concomitantly infected by both $H$. pylori and T. gondii, $100 \%$ suffered from severe gastritis with gastric erosions and ulcers; while $75 \%$ and $30.8 \%$ showed moderate and mild pathological signs respectively. The remaining mild gastritis cases (69.2\%) suffered from a single infection by either $H$. pylori or T. gondii. In consensus, it was reported that $H$. pylori antigen in stools was positive in $79 / 86$ of antral or corpus gastritis cases, $19 / 21$ of duodenitis cases, 2/2 of gastric ulcer cases, 8/8 of duodenal ulcer cases, 1/1 of gastric and duodenal ulcer cases and $17 / 21$ of esophagitis cases ${ }^{[23]}$. It appears that our report is the first record of the effect of toxoplasmosis on gastritis severity in humans. We recorded that in $H$. pylori negative cases, serum antiToxoplasma IgM was positive in $4 / 26$ cases showing mild antral erythematous mottling (mild cases); and serum anti-Toxoplasma IgG was positive in 6/26 cases showing antral gastritis with duodeno-gastric biliary reflux (mild cases); while in concomitant infections there were 8/26 mild cases, 12/16 moderate cases and $18 / 18$ severe cases of gastritis. In a recent case report of a patient complaining of odynophagia, dysphagia 
and dyspepsia, toxoplasmosis was involved alone as a zoonotic infection. Clusters of tachyzoites were discovered in histopathological sections of esophageal ulcers containing gastric inflammatory lymphocyte and eosinophil infiltrates ${ }^{[24]}$.

Another group of researches ${ }^{[25]}$ recorded examination of a gastric biopsy report from a-22-years old AIDS patient showing T. gondii infection of the stomach which appeared as intracellular trophozoites harboring the necrotic gastric epithelium, macrophages and muscle cells, in addition to the presence of true cysts and psuedocysts.

Our evaluation of the clinical significance of cag-A and vac-A antibodies in severe gastritis with gastric ulcers, showed weak expression in relation to mild and moderate gastritis only. On the other hand, the expression of these two $H$. pylori proteins, was supposedly related to the bacterium pathogenicity ${ }^{[26]}$. The researchers acknowledged that cag-A cytotoxin is involved in the development of peptic ulcer and is common in gastric adenocarcinoma, and that some subtypes of vac-A cytotoxin are considered as risk factors. Of note, in our study recurrent gastritis and gastric ulcers cases the $H$. pylori cag-A and vac- $A$ cytotoxins revealed high positivity $(71.4 \%$ and $57.1 \%$, respectively) proving their value as detectable markers for recurrence. Another study ${ }^{[18]}$ had revealed $H$. pylori infection in $51.4 \%$ of recurrent cases. This was advocated by other studies ${ }^{[27-30]}$.

Our study may be considered a new record for the role of anti-Toxoplasma IgM and IgG in naïve and recurrent cases of gastritis and gastric ulcers. Detection of IgM antibodies appeared more positive in recurrent cases being $57.1 \%$ versus $12.5 \%$ in naïve cases; while IgG antibodies were more positive in naïve cases being $81.3 \%$ versus $71.4 \%$ in recurrent cases. Therefore, both clinical conditions of naïve and recurring gastritis may be related to infection with $T$. gondii. It is interesting for us to note that in recurrent cases the mean anti-Toxoplasma IgM antibody was higher than in naïve cases, and vice versa the mean anti-Toxoplasma IgG in naïve cases was higher than in recurrent cases, indicating that the patients with gastric ulcer recurrence presented themselves earlier due to more acute symptoms, than those with chronic infections.

In conclusion, toxoplasmosis participates in recurrence of gastritis and gastric ulcers whether alone or in combination with H. pylori. Recurrence of gastric affection in toxoplasmosis patients is more common in acute infections. $H$. pylori cag-A is more frequent in recurrent cases. Recurrence is more obvious in the middle age, in males, and in those eating junk foods.

Author contributions: All authors participated in the study design. Elawamy WE, Ghazy AA and
Taha $\mathrm{AE}$ shared in performing all investigations, results interpretation, data analysis and writing the manuscript. Haydara T and Ghazy AA collected clinical samples and literature data. Haydara T performed the upper GIT endoscopy for all patients. Elawamy WE contributed to editing of the manuscript for publication. Competing interest: The authors declare no competing interests.

Funding statement: We received no funds for this research.

\section{REFERENCES}

1. Gale SD, Erickson LD, Brown BL, Hedges DW. Interaction between Helicobacter pylori and latent toxoplasmosis and demographic variables on cognitive function in young to middle-aged adults. PLoS One 2015; 10:e0116874.

2. Bui D, Brown HE, Harris RB, Oren E. Serologic evidence for fecal-oral transmission of Helicobacter pylori. Am J Trop Med Hyg 2016; 94:82-88.

3. Miwa H, Sakaki N, Sugano K, Sekine H, Higuchi K, Uemura $\mathrm{N}$, et al. Recurrent peptic ulcers in patients following successful Helicobacter pylori eradication: A multicenter study of 4940 patients. Helicobacter; 2004: 9:9-16.

4. Chang SS, Hu HY. Helicobacter pylori eradication within 120 days is associated with decreased complicated recurrent peptic ulcers in peptic ulcer bleeding patients. Gut Liver 2015; 9:346.

5. Stefano K, Marco M, Federica G, Laura B, Barbara B, Gioacchino L. Helicobacter pylori, transmission routes and recurrence of infection: State of the art. Acta Biomed 2018; 89:72.

6. Courtney E, Whary MT, Ihrig M, Bravo LE, Correa P, Fox JG. Serologic evidence that ascaris and Toxoplasma infections impact inflammatory responses to Helicobacter pylori in Colombians. Helicobacter 2012; 17:107-115.

7. Covacci A, Censini S, Bugnoli M, Petracca R, Burroni D, Macchia G, et al. Molecular characterization of the 128kDa immunodominant antigen of Helicobacter pylori associated with cytotoxicity and duodenal ulcer. Proc Natl Acad Sci USA 1993; 90:5791-5795.

8. Blaser MJ, Perez-Perez GI, Kleanthous H, Cover TL, Peek RM, Chyou P, et al. Infection with Helicobacter pylori strains possessing cagA is associated with an increased risk of developing adenocarcinoma of the stomach. Cancer Res 1995; 55:2111-2115.

9. Sobala G, Crabtree J, Dixon M, Schorah C, Taylor J, Rathbone B, et al. Acute Helicobacter pylori infection: clinical features, local and systemic immune response, gastric mucosal histology, and gastric juice ascorbic acid concentrations. Gut 1991; 32:1415-1418.

10. Keikha M, Ali-Hassanzadeh M, Karbalaei M. Association of Helicobacter pylori vacA genotypes and peptic ulcer in Iranian population: A systematic review and metaanalysis. BMC Gastroentrol 2020; 20(1):266. 
11. Glover M, Tang Z, Sealock R, Jain S. Diarrhea as a presenting symptom of disseminated toxoplasmosis. Case Rep Gastrointest Med 2017; 2017:3491087.

12. Buzoni-Gatel D, Schulthess J, Menard LC, Kasper LH. Mucosal defences against orally acquired protozoan parasites, emphasis on Toxoplasma gondii infections. Cell Microbiol 2006; 8:535-544.

13. Stoicov C, Whary M, Rogers AB, Lee FS, Klucevsek $\mathrm{K}, \mathrm{Li} \mathrm{H}$, et al. Coinfection modulates inflammatory responses and clinical outcome of Helicobacter felis and Toxoplasma gondii infections. J Immunol 2004; 173:3329-3336.

14. Gulcan EM, Varol A, Kutlu T, Cullu F, Erkan T, Adal E, et al. Helicobacter pylori stool antigen test. Indian J Pediatr 2005; 72:675-678.

15. Han FC, Li XJ, Jiang H, Qin LP, Li D, Guo YH, et al. Detection of $H$. pylori antibody profile in serum by protein array. World J Gastroenterol 2006; 12:40444048.

16. Balaghaleh MR, Farash BRH, Zarean M, Pourdehno $\mathrm{SH}$, Mirahmadi $\mathrm{H}$, Jarahi L, et al. Diagnosis of acute toxoplasmosis by IgG avidity method in pregnant women referred to health centers in south-eastern Iran. J Parasit Dis 2019; 43(3):517-521.

17. Ansari S, Yamaoka Y. Helicobacter pylori virulence factors exploiting gastric colonization and its pathogenicity. Toxins (Basel) 2019; (11):677.

18. Kim JJ, Kim N, Lee BH, Kang JM, Seo P, Lim MK, et al. Risk factors for development and recurrence of peptic ulcer disease. Korean J Gastroenterol 2010; 56:220228.

19. Kim N, Oh JH, Lee CG, Lim C, Won KH, Choi WR, et al. Effect of eradication of Helicobacter pylori on the benign gastric ulcer recurrence: A 24 month follow-up study. Korean J Intern Med 1999; 14(2):9-14.

20. Mahmood KT, Sara S, Shifa H. Eating habits a risk factor to peptic ulcer. J Pharm Sci Res 2011; 3(1):903-910
21. Gul S, Jawed L, Tariq S, Aziz S. Helicobacter pylori association with acid peptic disease: It's incidence in population having increase junk food intake. Transl Biomed 2016; 7:4.

22. Vomero ND, Colpo E. Nutritional care in peptic ulcer. Arq Bras Cir Dig 2014; 27:298-302.

23. Adiloglu AK, Isler M, Goren I, Candir O, Senol A, Onal $\mathrm{S}$, et al. Quantitative correlation of Helicobacter pylori stool antigen (HpSA) test with the severity of $H$. pylorirelated gastritis. Tohoku J Exp Med 2007; 212:159-167.

24. Lodenyo H, Rogena E, Sitati S. Oesophageal and gastric toxoplasmosis: Rare presentation of an emerging zoonotic disease. Afr J Health Sci 2019; 32:1-4.

25. Alpert L, Miller M, Alpert E, Satin R, Lamoureux E, Trudel L. Gastric toxoplasmosis in acquired immunodeficiency syndrome: ante-mortem diagnosis with histopathologic characterization. Gastroenterology 1996; 110:258-264.

26. Sokić-Milutinović A, Todorović V, Milosavljević T. Clinical significance of infection with cag $A$ and vac $A$ positive Helicobacter pylori strains. Srp Arh Celok Lek 2004; 132:458-462.

27. Maaroos HI, Kekki M, Vorobjova T, Salupere V, Sipponen P. Risk of recurrence of gastric ulcer, chronic gastritis, and grade of Helicobacter pylori colonization. A long-term follow-up study of 25 patients. Scand J Gastroenterol 1994; 29:532-536.

28. Sipponen P, Maaroos HI. Chronic gastritis. Scand J Gastroenterol 2015; 50:657-667.

29. Kosunen TU, Pukkala E, Sarna S, Seppälä K, Aromaa A, Knekt P, et al. Gastric cancers in Finnish patients after cure of Helicobacter pylori infection: A cohort study. Int J Cancer 2011; 128:433-439.

30. Lee YC, Chen TH, Chiu HM, Shun CT, Chiang H, Liu TY, et al. The benefit of mass eradication of Helicobacter pylori infection: A community-based study of gastric cancer prevention. Gut 2013; 62:676-682. 